\title{
Automated Computed Tomography Based Parasitoid Detection in Mason Bee Rearings
}

Bart R. Thomson ( $\square$ bart.thomson@usz.ch )

University Hospital of Zurich

Steffen Hagenbucher

Wildbiene + Partner AG

Robert Zboray

Swiss Federal Laboratories for Materials Science and Technology

Michelle Aimée Oesch

University of Zurich

Henning Richter

University of Zurich

\section{Research Article}

Keywords: Computer vision, Bee rearings, Parasitoid rate

Posted Date: November 15th, 2021

DOI: https://doi.org/10.21203/rs.3.rs-1048323/v1

License: (c) (1) This work is licensed under a Creative Commons Attribution 4.0 International License.

Read Full License 


\title{
Automated computed tomography based parasitoid detection in mason bee rearings
}

\author{
Bart R. Thomson ${ }^{1,2}$, Steffen Hagenbucher ${ }^{3}$, Robert Zboray ${ }^{4}$, Michelle Aimée \\ Oesch $^{5}$, and Henning Richter ${ }^{6}$ \\ ${ }^{1}$ Department of Neurosurgery, University Hospital and University of Zurich, Zurich, \\ Switzerland \\ ${ }^{2}$ Division of Internal Medicine, University Hospital Zurich, Schlieren, Switzerland \\ 3 Wildbiene + Partner AG, Zurich, Switzerland \\ ${ }^{4}$ Center for X-ray Analytics, EMPA, Dübendorf, Switzerland \\ ${ }^{5}$ Vetcom, University of Zurich, Zurich, Switzerland \\ ${ }^{6}$ Diagnostic Imaging Research Unit, University of Zurich, Zurich, Switzerland
}

\begin{abstract}
In recent years, insect husbandry has seen an increased interest in order to supply in the production of raw materials, food or as biological/environmental control. Unfortunately, large insect rearings are susceptible to pathogens, pests and parasitoids which can spread rapidly due to the confined nature of a rearing system. Thus, it is of interest to quickly and efficiently monitor the spread of such manifestations and the overall population size. Medical imaging techniques could be used for this purpose, as large volumes can be scanned non-invasively. Due to its 3D acquisition nature, computed tomography seems to be the most suitable for this task. This study presents an automated, computed tomography-based, counting method for bee rearings that performs comparable/similar to identifying all Osmia cornuta cocoons manually. The proposed methodology achieves this in an average of 7 minutes per sample, compared to 90 minutes per sample for the manual count over a total of 12 samples collected around lake Zurich in 2020. Such an automated bee population evaluation tool is a valuable in combating environmental influences on bee, and potentially other insect, rearings.
\end{abstract}

Keywords: Computer vision $\cdot$ Bee rearings $\cdot$ Parasitoid rate

\section{Introduction}

Animal husbandry has been an important part of human development as we heavily rely on animals for food and raw materials 7 . However, human effort was mostly focused on vertebrates, while insects (or other arthropods) were rarely reared and domesticated, with a few exceptions such as the silk moth or the honeybee 15. In recent years, there has been an increased interest in utilizing insects to satisfy human needs [15]. Mass rearing of insects for biological control, production of raw materials or as a food source is now common all over the globe and there are no signs that this trend is slowing down [15. Already the industry 
of apiculture has been shown to deliver at least 22 billion €to the European agricultural sector through pollination [6].

As with other animals, a large rearing of insects is susceptible to pathogens and pests [5]. Due to the confined nature of a rearing system, these can spread quickly and severely reduce yield or even destroy such a rearing. A unique threat to insect rearings are parasitoids. A parasitoid lives in close association with its host (either inside or on it) and, in contrast to a parasite, sooner or later kills its host [26]. Most parasitoids are Hymenoptera (so called parasitic wasps), but they are also found in other groups like the Dipterans. Normally a parasitoid lays its eggs inside a host, where the larvae emerge and consume the host [26]. Due to this lifestyle and their small size, most parasitoids are hard to detect and could remain undetected in a commercial rearing, until immense damage has been done [2. Female parasitic wasps can lay several hundred eggs and are very resistant towards inbreeding depressions [12, which means that even a small and isolated population can proliferate quickly.

With the decline of honeybee populations over the last decades [420], ensuring crop pollination has become a large concern 10 . Besides bumble bees, mason bees are an emerging beneficial arthropods that are used as managed pollinators in North America, Europe and East Asia 29]. They belong to the genus Osmia (Hymenoptera: Megachillidae) with four different species that are used (Osmia bicornis \& Osmia cornuta in Europe; Osmia cornifrons in Asia; Osmia lignaria in North America) 3[9]18].

Mason bees are so called solitary bees. They do not live in a social structure and do not have a queen or cast system. The above-mentioned species are univoltine and the adults are active in early spring [29. During these months the females build nests within suitable cavities. These nests are stocked with a mix of pollen and nectar and if the provision is large enough the bee will lay an egg on top. When the cavity is filled and the nest is finished, it is closed with a mud plug. After 3-7 days the larvae emerge from the egg and start consuming the pollen. The bees spend the summer as pupae in a diapause. In late summer, metamorphosis is completed where the bees remain within their cocoons and enter hibernation as adults. When spring temperatures are sufficiently high, bees will break their diapause and hatch. These adult mason bees have the potential to be an important pollinator of orchard crops, especially of early blooming plants like cherries and almonds [19]. They fly at low temperatures [34, are specialized on fruit trees 29] and have a small flight radius [35].

Rearing of these bees must be done outdoors, as it is impossible to provide enough flying space and floral resources in a greenhouse or a similar set-up. Thereby, the bees are constantly exposed to natural enemies. This increases to encounter of natural enemies drastically, as it is nearly impossible to prevent their entry into the breeding stock of mason bees. Some of these natural enemies could cause significant damage but are easily detected and removed with proper cocoon care [231]. This is however not possible for parasitic wasps that attack mason bees. The most destructive parasitoids in commercial mason bees rearings are Melittobia acasta (Hymenoptera: Eulophidae) and several species of 
Monodontomerus (Hymenoptera: Torymidae) 2231. These wasps lay their eggs inside the cocoons of the bees, the larvae consume the bee and overwinter inside the cocoons as larvae 1131. These parasitoids provide a significant challenge due to their concealed lifestyle, where the parasitoid population could increase undetected. Therefore, it is essential to monitor the abundance of parasitoids constantly. Monitoring manually by opening the cocoons is not feasible for a large number of bees and can potentially endanger the bee inside the cocoon.

A non-invasive method for parasitoid detection is the use of x-ray, which is a non-invasive imaging method that allows for examination of different aspects of mason bee biology [21/22 2324] (Fig. 1D). However, the use of x-ray radiography limits the amount of cocoons to be evaluated, as only one layer of cocoons which should be orientated on their longitudinal axis allows for evaluation of the parasitoids. X-ray radiography provides a two-dimensional image of a three-dimensional situation and the standardization of the cocoon orientation, while imaging large numbers, is impossible. Accordingly, conventional xray radiography is inadequate for assessment of a large-scale mason bee rearing. Alternatively, computed tomography (CT), a volumtric x-ray based technique, could be used. CT covers a three-dimensional volume and can therefore simultaneously scan multiple layers of cocoons. Accordingly, this results in a high throughput and the possibility for large-scale evaluations. CT based data allows to re-orientate the images in $3 \mathrm{D}$, independent of the original object orientation. This allows questionable cocoons to undergo a detailed evaluation and reduces error rate compared to x-ray radiography. Even if large data assessment is possible based on CT, the limited availability of accessible CT scanners and the need for manual counting still limits large-scale use in mason bee rearings.

In this study, we present a CT based approach for parasitoid detection in mason bee rearings. To our knowledge, this is the first time an automated screening method for a high number of cocoons is presented. Additionally, manual and automated counting of parasitoid rates are described and compared, providing incentive to up-scale future screenings.

\section{Results}

Conventional x-ray radiography and $\mathrm{CT}$ allowed to non-invasively distinguish between healthy bees and parasitoids inside the cocoons. X-ray radiography is applicable for individual cocoons or cocoons still inside their nesting substrate. Here, adult bees and parasitoids can be distinguished from each other (Fig. 1D), whereas $\mathrm{CT}$ allowed for volume rendering and multi-planar reconstruction (Fig. 1 A-C \& E). Mirco-CT renderings of parasitoids and a healthy bee are presented in Figure 2 together with macro photographs. These images clearly illustrate the anatomical differences between a healthy and parasitoid cocoon that are further analyzed by radiographical imaging. 


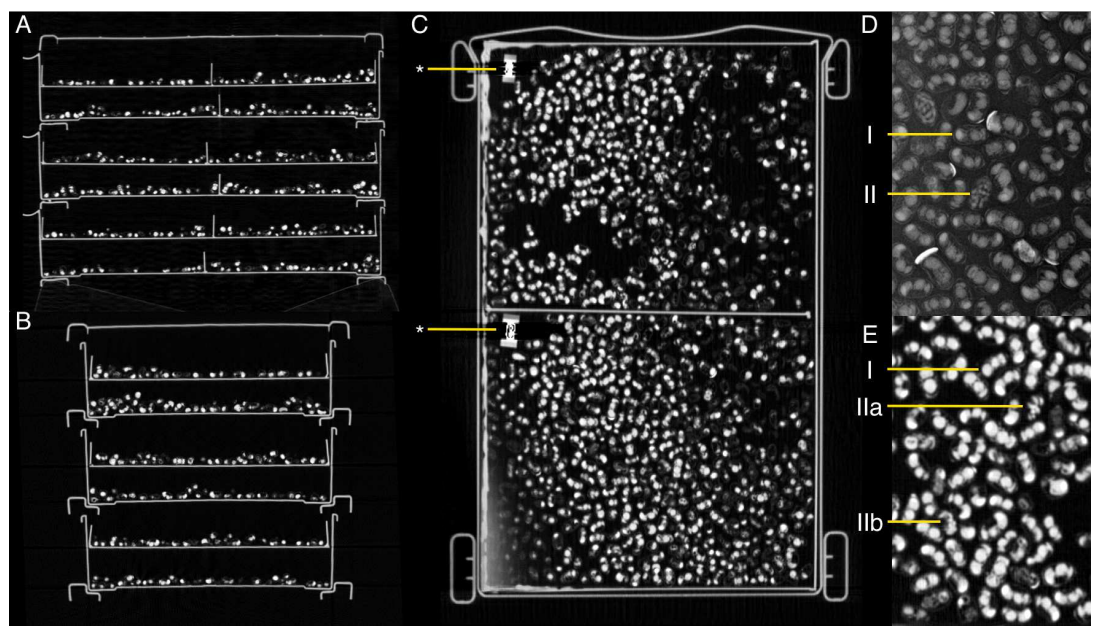

Fig. 1. CT scan of a customized plastic box containing around 6000 mason bees in 12 layers with metal numbers for identification of batches. A-C: Three dimensional view (latero-lateral, anterior-posterior, dorso-ventral), where the metal numbering is indicated with an *, D: Cocoon x-ray acquisition with healthy (I) and parasitoid (II) cocoons, E: Healthy (I) and two parasitoids which are easy (IIa) and challenging to detect (IIb), surrounded by adult bees in dorso-ventral view of CT acquisition. 

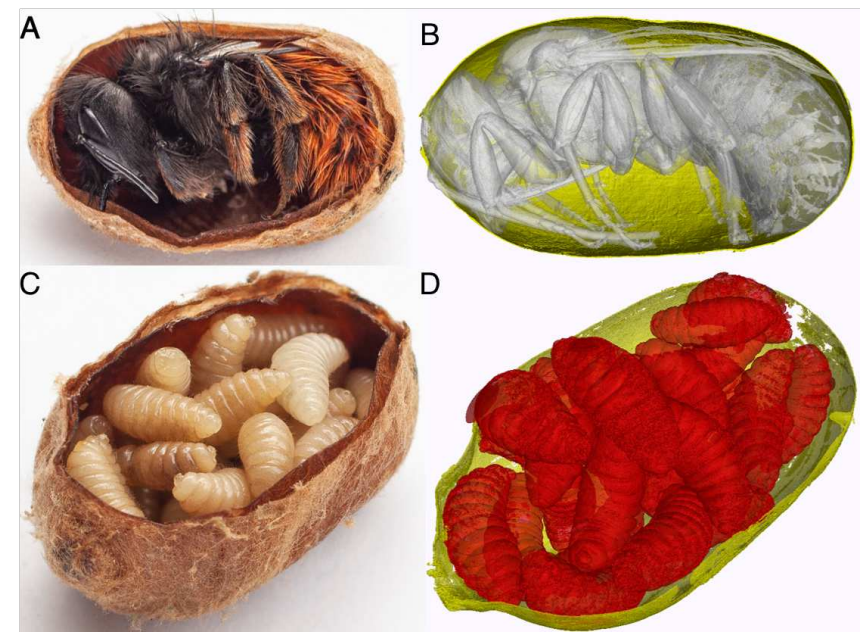

Fig. 2. Comparison of the incised healthy $(\mathbf{A})$ and parasitoid $(\mathbf{C})$ cocoons with $3 \mathrm{D}$ renderings and virtual incision based on high-resolution micro-CT results (B and $\mathbf{D})$. The high resolution of the micro-CT can enable non-invasive examination of the internal anatomy of a healthy bee in its cocoon. The larvae in the parasitoid cocoon can also be clearly resolved in details. Note that the larvae moved between the micro-CT and the preparation for photography inside the cocoon.

In 12 samples, a total of 5864 Osmia cornuta were manually counted within this study, according to the VC. Which is summarized in Table1, together with the RC of 3371 and the AC of 6394. All healthy cocoon countings were normally distributed, only the rapid manual counting was found to differ significantly $(p<0.001) .65$ parasitoids were found by $\mathrm{VC}$, comparing to respectively 21 and 48 in the RC and AC. Again, the difference between the $\mathrm{VC}$ and the RC was found to be significant $(p=0.004)$. All parasitoid cocoons identified by the AC were manually identified as true positives and no false positives were found. The aforementioned countings lead to parasitoid rates of $0.62 \%, 1.11 \%$ and $0.75 \%$ for the $\mathrm{RC}, \mathrm{VC}$ and $\mathrm{AC}$, respectively. Again, the RC and $\mathrm{VC}$ differed significantly $(p=0.039)$.

Counting times are reported as 4.5, 90 and 7 minutes per sample for the $\mathrm{RC}$, $\mathrm{VC}$ and $\mathrm{AC}$, respectively. However, image preparation time was not included in the $\mathrm{RC}$ processing time. 


\begin{tabular}{|c|c|c|c|c|c|c|c|c|c|}
\hline \multirow[t]{2}{*}{ Sample Number } & \multicolumn{3}{|c|}{ Healthy cocoons } & \multicolumn{3}{|c|}{ Parasitoid cocoons } & \multicolumn{3}{|c|}{ Parasitoid rate $(\%)$} \\
\hline & $\mathrm{RC}$ & $\mathrm{VC}$ & $\mathrm{AC}$ & $\mathrm{RC}$ & $\mathrm{VC}$ & $\mathrm{AC}$ & $\mathrm{RC}$ & VC & $\mathrm{AC}$ \\
\hline 1 & 221 & 301 & 341 & 0 & 2 & 1 & 0.0 & 0.66 & 0.29 \\
\hline 2 & 276 & 544 & 585 & 1 & 2 & 1 & 0.36 & 0.37 & 0.17 \\
\hline 3 & 362 & 690 & 714 & 0 & 3 & 1 & 0.0 & 0.43 & 0.14 \\
\hline 4 & 278 & 466 & 547 & 1 & 2 & 2 & 0.36 & 0.43 & 0.37 \\
\hline 5 & 268 & 378 & 413 & 2 & 6 & 6 & 0.75 & 1.59 & 1.45 \\
\hline 6 & 301 & 591 & 625 & 1 & 2 & 1 & 0.33 & 0.34 & 0.16 \\
\hline 7 & 279 & 466 & 513 & 8 & 18 & 17 & 2.87 & 3.86 & 3.31 \\
\hline 8 & 260 & 553 & 551 & 3 & 8 & 6 & 1.15 & 1.45 & 1.09 \\
\hline 9 & 334 & 402 & 467 & 0 & 7 & 3 & 0.0 & 1.74 & 0.64 \\
\hline 10 & 292 & 520 & 589 & 0 & 3 & 1 & 0.0 & 0.58 & 0.17 \\
\hline 11 & 216 & 407 & 432 & 2 & 2 & 2 & 0.93 & 0.49 & 0.46 \\
\hline 12 & 284 & 546 & 617 & 3 & 10 & 7 & 1.06 & 1.83 & 1.13 \\
\hline Total & $3371^{*}$ & 5864 & 6394 & $21^{*}$ & 65 & 48 & $0.62^{*}$ & 1.11 & 0.75 \\
\hline
\end{tabular}

Table 1. 12 samples of Osmia cornuta by Monodontomerus spp., in bees recovered around lake Zurich (2020) and their countings of identified healthy and parasitoid cocoons for the rapid (RC), volumetric (VC) and automated (AC) method. The ground truth, VC, is indicated in bold.

\section{Discussion}

Detection of parasitoids within a mason bee rearing remains a challenge when rearing these bees on a large commercial scale, we have shown that parasitoids inside cocoons can be quantified by the use of x-ray imaging techniques. Conventional x-ray radiography provides limited space on the plates as they are available in three dimensions: small $18 \times 24 \mathrm{~cm}$, medium $24 \times 30 \mathrm{~cm}$ and large $35 \times 42$ $\mathrm{cm}$, therefore limiting the number of cocoons per x-ray acquisition. Accordingly, numerous $\mathrm{x}$-rays acquisitions would be needed to analyze a representative subpopulation of the bee rearing. Where micro-CT allows scanning of small volumes with very high resolution for detailed analysis, commercially available clinical CT scanners provide a scan volume for an uncountable number of bees with a sufficiently accurate image quality to distinguish healthy from parasitoid cocoons. To our knowledge, this is the first study that indicates CT as a modality of choice for up-scaling the screening of parasitoids within large batches of mason bee rearings.

Mason bee rearings that would like to profit from these techniques in the future, could use an x-ray system or organize access to CT scans. The first option requires expertise in radiation protection as well as sufficient funding, but limits efficiency of screenings. The second option offers all for a large-scale data acquisition, but is limited by the availability of accessible CT scanners. Even if the number of clinical CT scanners is still increasing in human and veterinary medicine, accessibility in accordance with hygiene requirements of the corresponding institute/clinic remains a limiting factor. Whenever access 
to $\mathrm{CT}$ imaging is acquired, the overall aim will be to maximize outcome per scan duration. Therefore, it becomes important to up-scale the scanned sample size whilst at the same time shortening examination time. While CT imaging of large-scale batches can be acquired time efficiently, the analysis of the resulting dataset is tedious, time and resources consuming as well as prone to errors.

In this study, it was challenging to get all cocoons into focus simultaneously with the rapid manual counting, as single CT slices were chosen. Accordingly, for some samples multiple slices had to be chosen for counting, resulting in an uncertainty to either double count cocoons due to ambiguous positioning, or not at all. When comparing this $\mathrm{RC}$ to the $\mathrm{VC}$, almost double the amount of cocoons are identified in the VC. However, the estimated parasitoid rate gives an approximation of the parasitoid rate as the amount of found parasitoids is also roughly half of the VC. A major downside is that it would take an inexperienced observer more time and most likely result in less accurate counting. Based on traditional image processing techniques, our automated approach has shown a significant time improvement when compared to $\mathrm{VC}$, whilst obtaining more accurate results than RC. Unfortunately, analysis of CT data does not allow identification of the parasitoid species; experience suggests that the underlying parasitoids belong to the genus Monodontomerus, where the most common parasitoids of mason bees are found 31.

Given these promising results, future work should focus on novel image processing techniques such as deep learning to assist or replace subjective analysis and enable higher throughput and accuracy. These methodologies are already applied extensively and accurately in, amongst others, the field of radiology in human medicine [16. For example, the potential of segmenting smaller structures in CT volumes is successfully presented in radiological literature [17/28]. These techniques could eliminate the need for a custom made box and potentially allow for scanning of a collection of cocoons in any arbitrary structure, further decreasing the time and effort spent in scan acquisition. Additionally, a low x-ray absorption material such as styropor could be used for the custom made box, as this will reduce the amount of noise.

Moreover, additional imaging can be used to further differentiate contents of either healthy or parasitoid cocoons. For this purpose a healthy and a parasitoid cocoon were scanned by micro-CT, followed by comparing its 3D segmentation to classical macro photography, after dissection of these cocoons. The highresolution provided by micro-CT allows to identify underlying parasitoid species or to visualize mason bee anatomy details as well as to measure their structures on a micrometer level. Furthermore, micro-CT as a non-invasive imaging method is compatible with the potential research need of intact structures and is tackling research questions needing higher resolution. A drawback of micro-CT imaging is the limited sample size as the resolution decreases when increasing sample size. Moreover, due to its long scan duration it is more prone to movement artifacts, when compared to clinical CT. This prevents the use of micro-CT for commercial large-scale batch analysis but it remains a useful complementary tool. 
Despite the low percentage in populations from different origins, the presence of detected parasitoids shows the potential risk they can cause. An inadequately managed mason bee rearing, could suffer high loss by these parasitoids. Therefore, well-managed mason bee rearings should include a method to detect parasitoids [2]. Moreover, the monetary value of pollination services has been estimated at 22 billion $€$, in Europe [6], suggesting future potential for such detection methods. Without early detection methods, the infestation would only be detected when significant damage has been caused. Combined with declines in bee populations due to climate change [30], this could be disastrous for the proliferation of bee rearings. While it is impossible to screen all cocoons, sampling of a representative sub-population would allow for detection of an accumulation of bee numbers over several years and stimulate the development of measures to counter that. The optimal size for such a sub-population needs to be determined in future research.

While our study did not detect large differences between samples, suggesting an even parasitoid load, there are several additional applications of these techniques. As parasitoid rates are often linked to factors such as climate [13], vegetation 8 , or habitat fragmentation [32, studies could be designed to better understand the effect of its exposure to mason bees. This can also be applied to other fields of ecological research to better understand the population dynamics of parasitoids with a hidden lifestyle, that is not easily identified by visual inspections.

\subsection{Conclusion}

The results of this study show an improved non-invasive parasitoid screening inside cocoons of mason bees and could potentially be extrapolated to other insect rearings. X-ray based imaging methods such as x-ray, CT and micro-CT enable to non-invasively differentiate between healthy and parasitoid cocoons in mason bee rearings. Furthermore, CT allows an efficient and effective screening for parasitoids, especially in large-scale batches. The automated counting surpasses rapid manual counting and could possibly be further improved by deep learning techniques. Parasitoid rate was low within the randomly chosen samples and did not show significant differences. Influence of mason bee species, environmental factors, climate and geographical differences on these results has to be shown in future research.

\section{Materials and Methods}

All samples examined for this study were provided by the company 'Wildbiene + Partner AG' (Zurich, Switzerland). The European mason bee species Osmia cornuta (Hymenoptera: Megachillidae) was used as a model organism. Imaging was conducted with bees that developed in 2020 and were reared around Lake Zurich. The bees were brought to the rearing sites in mid-March and were allowed to nest. With the adults dying, flight activity of the bees stopped in 
late April, while their offspring developed inside the nesting substrate (Arundo Donax, Giant reed stalks). In late August the bees had mostly finished their development into adults and the nesting material was collected and the cocoons were removed from it. In February 2021, the bees were imaged.

Imaging for this study was conducted at the Diagnostic Imaging Research Unit at the Vetsuisse Faculty of the University Zurich. First trails for imaging parasitic wasps inside the cocoon of mason bees included conventional x-ray radiography. All x-rays were generated with the digital x-ray system (Fujifilm CR, Profect / Capsula CS), and the digital picture archiving system (PACS Intellispace Philips).

Computer tomography (CT) was performed using a Siemens Brilliance 16 slice CT scanner (slice thickness 0.8mm, KVP 120, mAs 151, spacing between slices $0.4 \mathrm{~mm}$ ) with a bone and soft tissue reconstruction protocol. The randomly selected mason bee batch samples were scanned as a multiple layer volume in a custom-made box containing 12 compartments, each holding a maximum of around 400 cocoons of $\mathrm{O}$. cornuta (Fig $3 \mathrm{~A})$. The cocoons were scanned in single layers marked with metal numbers, which facilitated discriminating between samples for manual counting (Fig $3 \mathrm{C}$ ). Additionally, as a pre-processing step for the automated counting, the same custom-made box was scanned without cocoons.
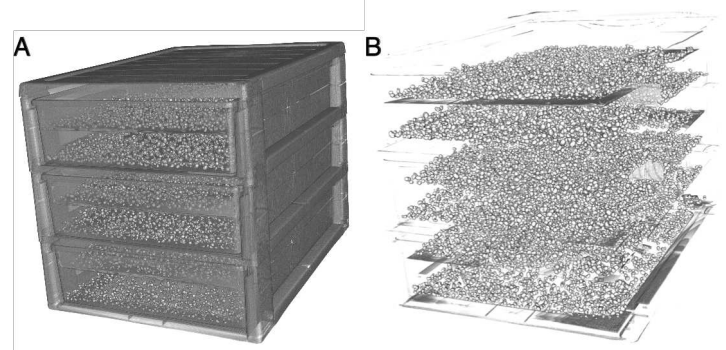

C

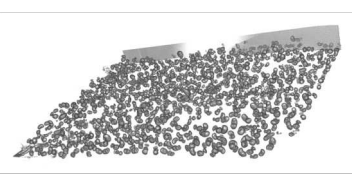

Fig. 3. 3D volume visualizations prior to AC. A: 3D box filled with cocoons, B: Visualization of cocoons after subtraction of the empty box once registered to the full box, C: Visualization of an individual sample.

\subsection{Rapid and volumetric manual count}

All DICOM images were assessed with the freeware Synedra View Personal (Version 19.0.0.2; Synedra Information Technologies) on a Windows computer system or Horos (Horos Project, 64-bit medical image viewer for OS X, GNU Lesser General Public License, Version 3 (LGPL-3.0) on a Macintosh computer system. A multi-planar reconstruction (MPR) of each dataset was performed and allowed re-orientation and image alignment in all dimensions. Assessment of healthy and parasitoid cocoons was performed manually by two different approaches: 
- After exporting suitable images into ImageJ [27, an experienced observer discriminated the sub populations by manual counting of several slices, which is coined rapid manual counting $(\mathrm{RC})$.

- Another experienced observer performed a true manual counting in the complete 3D volume, coined volumetric manual counting (VC). Here, all cocoons present in the volume where individually identified using 3D Slicer [25].

Processing time for both techniques was recorded (without image preparation) to allow for comparison with the automated counting method.

\subsection{Automated counting}

Prior to fully automated counting (AC), the regions of interest (ROIs) for each of the separate 12 compartments were manually identified using 3D Slicer [25]. Prior to counting of the individual ROIs, the scan of the empty custom-made box was registered to the scan which contained the cocoons based on the non-rigid method of Mattes et al. 19. The registered empty box volume was subtracted from the cocoon-filled box, resulting in a box-filtered volume (Fig. $3 \mathrm{~B}$ ) where additional noise, due to imperfect overlap of the two volumes, was removed by eroding and dilating operations. A threshold of -700 Hounsfield Units (HU), followed by morphological opening and dilating led to the final volume used for counting. First, all cocoons were identified based on a watershedding algorithm with a minimal size of 10 voxels and an inter component distance smaller than 1 voxel. Second, all identified cocoons that were $150 \%$ larger than the median cocoon size were split by a threshold of $-550 \mathrm{HU}$ followed by a dilation operation. An additional increase in threshold to $-450 \mathrm{HU}$, followed by a dilation operation was applied to the cocoons that were still identified as $150 \%$ larger than the median cocoon size after the aforementioned processing.

To differentiate between healthy and parasitoid cocoons, mean histograms of typical parasitic and healthy cocoons were acquired from an independent sample. The histogram profiles of all individual cocoons were iteratively compared with these mean histograms, allowing the algorithm to eliminate obvious healthy cocoons. Consecutively, all remaining cocoons that had an intensity variance lower than 80.000 were split by a threshold $300 \mathrm{HU}$ lower than the maximum HU value in the individual cocoon. In case of a parasitic cocoon, this allowed for identification of the individual parasitoids, whereas healthy cocoons would only present their hyper-intense hindbody and head. Separate hyper-intensities, based on the water shedding approach as reported in the foregoing paragraph, allowed classification of cocoons as parasitic when more than 6 hyper-intensities were present. All computation was performed in Python 33 .

\subsection{Micro-CT}

Additional micro-CT scans were conducted on manually selected individual cocoons at the Center for x-ray Analytics of EMPA (Dübendorf, Switzerland) to 
visualize differences in parasitoid and healthy cocoons in detail. For x-ray microCT, an EasyTom XL Ultra 230-160 micro/nano-CT scanner was used (RxSolutions SAS, Chavanod, France). The scanner features a Hamamatsu open, reflection micro-focus x-ray tube and a Varian PaxScan 2520DX detector (flat panel with amorphous silicon and a CsI conversion screen; 1920 x 1536 pixel matrix; pixel pitch of $127 \mathrm{~mm}$; 16 bits of dynamic range). As no predefined protocols exist on such micro-CT scanner for soft biosamples, expert judgement was used for defining the scan parameters. The tube was operated at $70 \mathrm{kV}$ and a current of $150 \mathrm{~mA}$. The voxel size of the CT scan was $8.3 \mu \mathrm{m}$ for the parasitoid and $9.1 \mu \mathrm{m}$ for the healthy cocoon. The images were acquired at 5.0 frames per second, averaging 2 frames per projection. The healthy bees in the cocoons and especially the larvae in the parasitoid cocoons tended, even if they were stored at very low temperatures prior to scanning, to move during scanning. This, at the high resolution of the micro-CT, potentially results in motion artefacts. To minimize the chance of motion during the scan, the scan time was reduced to a minimum whilst still maintaining acceptable image quality.

\subsection{Macro photography of specimen}

A focal length of $50 \mathrm{~mm}$ was chosen to obtain a close photographic representation of the visually perceived specimen. Two studio flashes were set indirectly to the specimen resulting in emitting soft light, avoiding harsh shadows and distracting highlights. Five to fifteen single images with individually set focus points were manually merged in post-production with Adobe Photoshop CC 2021, to enable a greater depth of field, enhancing the visibility of the specimen.

\subsection{Statistics}

Statistical evaluation of the data was performed with the Python library scipy [14. Here, the Shapiro-Wilk test was used to investigate normality, followed by significance testing in the healthy cocoons by an independent samples ttest. Whereas the parasitoid cocoons and the parasitoid rate were tested by the Wilcoxon rank sum test for 2 independent measurements.

\subsection{Acknowledgements}

The authors do not have any acknowledgements to make.

\subsection{Competing interests}

The authors do not declare any compete of interests.

\subsection{Author contribution}

BT Analysed the data and wrote the paper, $\mathrm{SH}$ wrote the paper and gathered the data, RZ performed measurements and wrote the paper, MAO performed measurements and HR designed the study, performed measurements, annotated data and wrote the paper. BT and SH share first authorship. 


\section{References}

1. Bosch, J., Blas, M.: Foraging behaviour and pollinating efficiency of osmia cornuta and apis mellifera on almond (hymenoptera, megachilidae and apidae). Applied Entomology and Zoology 29(1), 1-9 (1994)

2. Bosch, J., Kemp, W.P.: How to manage the blue orchard bee. Sustainable Agriculture Network, Beltsville, MD (2001)

3. Bosch, J., Kemp, W.P., Peterson, S.S.: Management of osmia lignaria (hymenoptera: Megachilidae) populations for almond pollination: methods to advance bee emergence. Environmental Entomology 29(5), 874-883 (2000)

4. Chauzat, M.P., Cauquil, L., Roy, L., Franco, S., Hendrikx, P., Ribière-Chabert, M.: Demographics of the european apicultural industry. PloS one 8(11), e79018 (2013)

5. Cohen, A.C., et al.: Ecology of insect rearing systems: a mini-review of insect rearing papers from 1906-2017. Advances in Entomology 6(02), 86 (2018)

6. Deloitte: Evaluation of Measures for the Apiculture Sector. https://op.europa. eu/s/pBvn (2013), [Online; accessed 8-July-2021]

7. Diamond, J.: Evolution, consequences and future of plant and animal domestication. Nature 418(6898), 700-707 (2002)

8. Durocher-Granger, L., Mfune, T., Musesha, M., Lowry, A., Reynolds, K., Buddie, A., Cafà, G., Offord, L., Chipabika, G., Dicke, M., et al.: Factors influencing the occurrence of fall armyworm parasitoids in zambia. Journal of Pest Science pp. $1-14(2020)$

9. Eeraerts, M., Vanderhaegen, R., Smagghe, G., Meeus, I.: Pollination efficiency and foraging behaviour of honey bees and non-apis bees to sweet cherry. Agricultural and Forest Entomology 22(1), 75-82 (2020)

10. Gallai, N., Salles, J.M., Settele, J., Vaissière, B.E.: Economic valuation of the vulnerability of world agriculture confronted with pollinator decline. Ecological economics 68(3), 810-821 (2009)

11. Glasser, S., Farzan, S.: Host-associated volatiles attract parasitoids of a native solitary bee, osmia lignaria say (hymenoptera, megachilidae). Journal of Hymenoptera Research 51, 249 (2016)

12. Henter, H.J.: Inbreeding depression and haplodiploidy: experimental measures in a parasitoid and comparisons across diploid and haplodiploid insect taxa. Evolution 57(8), 1793-1803 (2003)

13. Jeffs, C.T., Lewis, O.T.: Effects of climate warming on host-parasitoid interactions. Ecological Entomology 38(3), 209-218 (2013)

14. Jones, E., Oliphant, T., Peterson, P., et al.: SciPy: Open source scientific tools for Python (2001-), http://www.scipy.org/

15. Lecocq, T.: Insects: the disregarded domestication histories (2018)

16. van Leeuwen, K.G., Schalekamp, S., Rutten, M.J., van Ginneken, B., de Rooij, M.: Artificial intelligence in radiology: 100 commercially available products and their scientific evidence. European radiology 31(6), 3797-3804 (2021)

17. Li, X., Gong, Z., Yin, H., Zhang, H., Wang, Z., Zhuo, L.: A 3d deep supervised densely network for small organs of human temporal bone segmentation in ct images. Neural Networks 124, 75-85 (2020)

18. Matsumoto, S., Abe, A., Maejima, T.: Foraging behavior of osmia cornifrons in an apple orchard. Scientia horticulturae 121(1), 73-79 (2009)

19. Mattes, D., Haynor, D.R., Vesselle, H., Lewellyn, T.K., Eubank, W.: Nonrigid multimodality image registration. In: Medical Imaging 2001: Image Processing. vol. 4322, pp. 1609-1620. International Society for Optics and Photonics (2001) 
20. Meixner, M.D., et al.: A historical review of managed honey bee populations in europe and the united states and the factors that may affect them. Journal of invertebrate pathology 103, S80-S95 (2010)

21. Müller, A., Prosi, R., Praz, C., Richter, H.: Nesting in bark-the peculiar life history of the rare boreoalpine osmiine bee osmia (melanosmia) nigriventris (hymenoptera, megachilidae). Alpine Entomology 3, 105 (2019)

22. Müller, A., Prosi, R., Taylor, S., Richter, H., Herrmann, M., Weibel, U.: Unique nesting biology of osmia (melanosmia) uncinata, a palaearctic osmiine bee specialized on thick-barked conifers (hymenoptera, megachilidae). Alpine Entomology 4, 157-171 (2020)

23. Müller, A., Richter, H.: Dual function of potentilla (rosaceae) in the life history of the rare boreoalpine osmiine bee hoplitis (formicapis) robusta (hymenoptera, megachilidae). Alpine Entomology 2, 139-147 (2018)

24. Müller, A., Richter, H.: Neststandort und nestarchitektur der in leeren schneckengehäusen nistenden mauerbiene osmia andrenoides (hymenoptera, megachilidae). Entomo Helvetica 12, 69 (2019)

25. Pieper, S., Halle, M., Kikinis, R.: 3d slicer. In: 2004 2nd IEEE international symposium on biomedical imaging: nano to macro (IEEE Cat No. 04EX821). pp. 632-635. IEEE (2004)

26. Quicke, D.L., et al.: Parasitic wasps. Chapman \& Hall Ltd (1997)

27. Rasband, W.S., et al.: Imagej (1997)

28. Ren, X., Xiang, L., Nie, D., Shao, Y., Zhang, H., Shen, D., Wang, Q.: Interleaved 3d-cnn s for joint segmentation of small-volume structures in head and neck ct images. Medical physics 45(5), 2063-2075 (2018)

29. Sedivy, C., Dorn, S.: Towards a sustainable management of bees of the subgenus osmia (megachilidae; osmia) as fruit tree pollinators. Apidologie 45(1), 88-105 (2014)

30. Soroye, P., Newbold, T., Kerr, J.: Climate change contributes to widespread declines among bumble bees across continents. Science 367(6478), 685-688 (2020)

31. Stanisavljević, L.: The accompanying fauna of osmia cornuta and osmia rufa and effective measures of protection. Bulletin of Insectology 58(2), 141-152 (2005)

32. Tscharntke, T., Brandl, R.: Plant-insect interactions in fragmented landscapes. Annual Reviews in Entomology 49(1), 405-430 (2004)

33. Van Rossum, G., Drake Jr, F.L.: Python tutorial, vol. 620. Centrum voor Wiskunde en Informatica Amsterdam (1995)

34. Vicens, N., Bosch, J.: Weather-dependent pollinator activity in an apple orchard, with special reference to osmia cornuta and apis mellifera (hymenoptera: Megachilidae and apidae). Environmental Entomology 29(3), 413-420 (2000)

35. Zurbuchen, A., Landert, L., Klaiber, J., Müller, A., Hein, S., Dorn, S.: Maximum foraging ranges in solitary bees: only few individuals have the capability to cover long foraging distances. Biological Conservation 143(3), 669-676 (2010) 